\title{
Cell-cycle arrest at G2/M and proliferation inhibition by adenovirus-expressed mitofusin-2 gene in human colorectal cancer cell lines
}

\author{
X. CHENG' ${ }^{1}$ D. ZHOU 2 , J. WEI' ${ }^{2}$ J. LIN ${ }^{1, *}$ \\ ${ }^{1}$ Department of Colorectal Surgery, First Affiliated Hospital, Zhejiang University School of Medicine, Hangzhou, Zhejiang, 310003, China; ${ }^{2}$ Key \\ Lab of Combined Multi-organ Transplantation, Ministry of Public Health, Key Lab of Organ Transplantation, First Affiliated Hospital, Zhejiang \\ University School of Medicine, Hangzhou, China
}

*Correspondence: xf5923@126.com

Received December 8, 2012 / Accepted April 8, 2013

\begin{abstract}
The mitofusin-2 (Mfn2) is a novel gene characterised as a cell proliferation inhibitor. Mfn2 protein over-expression, mediated by an adenovirus, has a significant anti-proliferative effect in hepatoma carcinoma cells. However, there is no report on the effect of Mfn2 on colorectal cancer (CRC). In this study, we found that Mfn2 protein and mRNA levels were downregulated in CRC tissues compared to nearby normal tissues. An adenovirus encoding the complete Mfn2 open reading frame (Ad-Mfn2) exhibited a prominent anti-proliferative effect in the CRC cell lines HCT 116, HT-29, and SW480. AdMfn2 infection significantly inhibited the proliferation of CRC cells compared with Ad-GFP infection. Mfn2 overexpression resulted in a cell-cycle arrest at G2/M phase in CRC cells, and it increased the levels of p-cdc2 (Tyr15) and Myt1; however, the levels of cyclin B1 and p-ERK1/2 was reduced. Mfn2 overexpression aslo increased the levels of active caspase-3 and cleaved PARP. Taken together, these findings indicate that Mfn2 has a significant anti-proliferative effect in CRC cells using adenoviral vectors.
\end{abstract}

Key words: mitofusin-2(Mfn2), anti-proliferative, cell-cycle, G2/M phase, colorectal cancer (CRC)

Colorectal cancer (CRC) is the third most common malignancy and the second leading cause of cancer deaths worldwide [1]. CRC arises through a multi-step, multimechanism process consisting of initiation, promotion, and progression. Patients with CRC usually present with a change in bowel habits, rectal bleeding, abdominal pain, or weight loss [2]; however, most cases are diagnosed when the cancer is fairly advanced. Some genes and their expressed proteins, including APC, CTNNB1, KRAS2, BRAF, MADH4/SMAD4, TP53, PIK3CA, and TGFBR2, have been investigated with regards to the initiation and development of CRC [3-4]. Understanding the molecular basis of CRC will facilitate diagnosis and treatment. So it is necessary to find out more genes associated with initiation and development of CRC.

The mitochondrial GTPase mitofusin-2 (Mfn2), which is also known as hyperplasia suppressor gene (HSG), was first characterised by Chen et al. [5] as a suppressor of cell proliferation. Recent studies have shown that Bax activates the assembly of Mfn2 GTPase by interacting with the Mfn2 coiled-coil domain and co-localising with Mfn2 during apoptosis [6-7].
The anti-proliferative effect of Mfn2 is comparable to that of the well-known tumor suppressor p53 [5, 8]. We demonstrated previously that Mfn2 is a novel p53-inducible target gene in hepatocellular carcinoma cells [9].

Previous study showed that Mfn2 is involved in apoptosis and has anti-proliferative effect in hepatocellular carcinoma (HCC) [10]; however, it is unclear whether Mfn2 participates in apoptosis or has anti-proliferative effect in CRC. In this study, we assessed Mfn2 expression in CRC tissues and nearby normal tissues. Furthermore, we studied the effect of Mfn2 on CRC cell growth through the transfection of an adenovirus vector encoding Mfn2.

\section{Materials and methods}

Tissue samples, cell lines, and cell culture. Fifteen pairs of samples were obtained from the CRCs and adjacent benign colon tissues (more than $5 \mathrm{~cm}$ from the tumor margin) of patients who underwent surgical treatment for CRC at First Affiliated Hospital, College of Medicine, Zhejiang University. 
The CRC cell lines HCT 116, HT-29, and SW480 preserved at our institute were cultured in RPMI media 1640 supplemented with $10 \%$ foetal bovine serum (FBS) and $100 \mathrm{U} / \mathrm{mL}$ penicillin/streptomycin. All cells were maintained under a humidified atmosphere containing $5 \% \mathrm{CO}_{2}$ at $37^{\circ} \mathrm{C}$ and were passaged using standard cell culture techniques.

Virus construction and adenovirus infection. Replication-defective adenoviruses encoding the complete Mfn2 open reading frame (Ad-Mfn2) and a control adenoviral vector encoding green fluorescent protein (Ad-GFP) were constructed by Vector Gene Technology Company Ltd. (Beijing, China). Cell synchronisation was achieved by culturing the cells in RPMI media 1640 containing $0.2 \%$ FBS for $24 \mathrm{~h}$. The cells were then incubated with adenovirus at a multiplicity of infection of $100 \mathrm{pfu} / \mathrm{cell}$ in a small volume of serum-free medium. Following adsorption for $4 \mathrm{~h}$, the adenovirus-containing medium was discarded, fresh complete growth medium was added, and the cells were cultured in preparation for the following experiments. Nearly $90 \%$ of the cells were GFP-positive 24 $\mathrm{h}$ after infection.

Reverse transcription-polymerase chain reaction (RT-PCR) analysis. Mfn2 mRNA expression was detected by RT-PCR. Tissues were collected, and total RNA was extracted using TRIZOL reagent (Invitrogen, Carlsbad, CA, USA) according to the manufacturer's protocol. Total RNA concentration and quantity were assessed by absorbency at $260 \mathrm{~nm} / 280 \mathrm{~nm}$ using a DNA/Protein Analyzer (UV-2450, Shimadzu, Tokyo, Japan). RT was performed in a $25-\mu$ l reaction volume with $3 \mu \mathrm{g}$ of total RNA and treated with M-MLV reverse transcriptase to synthesise first-strand cDNA (Promega, Madison, WI, USA) according to the manufacturer's recommendations, followed by cDNA amplification using specific primer sets for Mfn2 and $\beta$-actin as an internal control. The primers used were: 5'-GGCAGCACATGGAGCGTTGT-3' (forward) and5'TGCAATCTGCTTGGCCCGGA-3' (reverse) for Mfn2, and 5'-CTTAGTTGCGTTACACCCTTTC-3' (forward) and 5'-CACCTTCACCGTTCCAGTTT-3' (reverse) for $\beta$-actin. The resulting products were 286 and $151 \mathrm{bp}$ in length. The amplification of Mfn2 was achieved as follows: one cycle of $94^{\circ} \mathrm{C}$ for $4 \mathrm{~min}$ followed by 32 cycles of $94^{\circ} \mathrm{C}$ for $45 \mathrm{~s}, 60^{\circ} \mathrm{C}$ for $45 \mathrm{~s}$, and $72^{\circ} \mathrm{C}$ for $45 \mathrm{~s}$, with a one-cycle extension at $72^{\circ} \mathrm{C}$ for $10 \mathrm{~min}$. The amplification of $\beta$-actin was achieved as follows: one cycle of $94^{\circ} \mathrm{C}$ for $4 \mathrm{~min}$ followed by 30 cycles of $94^{\circ} \mathrm{C}$ for $30 \mathrm{~s}, 57^{\circ} \mathrm{C}$ for $30 \mathrm{~s}$, and $72^{\circ} \mathrm{C}$ for $1 \mathrm{~min}$, with a one-cycle extension at $72^{\circ} \mathrm{C}$ for $10 \mathrm{~min}$. All products were separated by $1.5 \%$ agarose gel electrophoresis and visualised with ethidium bromide staining.

Western blot analysis. Infected cells were given fresh, complete growth medium and incubated for $48 \mathrm{~h}$. The tissues were homogenised in liquid nitrogen. Total protein was isolated from the cells and tissues using lysis buffer (Cell

Signalling Technology, Danvers, MA, USA) and subjected to Western blotting as described previously [10]. The primary antibodies were: anti-Mfn2 (1:1,000; Sigma, St. Louis, MO, USA); anti-p21 (1:1,000; Cell Signalling Technology); anti-cleaved caspase-3 (1:1,000; Cell Signaling Technology); anti-cleaved PARP (1:1,000; Cell Signalling Technology); anti-phosphocdc2 (1:1,000; Cell Signalling Technology); anti-cyclin B1 (1:1,000; Cell Signalling Technology); anti-Myt1 (1:1,000; Cell Signalling Technology); anti-phospho-ERK1/2 (1:1,000; Cell Signalling Technology); anti-phospho-Akt (1:1,000; Cell Signalling Technology); and anti- $\beta$-actin (1:1,500; Sigma).

Cell viability assay. Cell proliferation after infection with the various adenoviral vectors was assessed using WST-8 dye (Dojindo, Kyushu, Japan) according to the manufacturer's instructions. Briefly, $5 \times 10^{3}$ cells/well were seeded in a 96-well flat-bottomed plate, grown at $37^{\circ} \mathrm{C}$ for $24 \mathrm{~h}$, and incubated under serum-starved conditions for an additional $6 \mathrm{~h}$. Subsequently, the cells were treated with adenoviral vector in the presence of $10 \% \mathrm{FBS}$ for 24,48 , and $72 \mathrm{~h}$. Next, $10 \mu \mathrm{l}$ of WST-8 dye was added to each well, the cells were incubated at $37^{\circ} \mathrm{C}$ for $1 \mathrm{~h}$, and the absorbance was measured at $450 \mathrm{~nm}$.

Flow cytometric cell-cycle analysis. HCT 116, HT-29, and SW480 cells were seeded in 60-mm dishes in RPMI media 1640 plus 10\% FBS. After $12 \mathrm{~h}$, the medium was changed to RPMI media 1640 plus 5\% FBS without (mock) or with AdGFP or Ad-Mfn2. The cells were collected at $48 \mathrm{~h}$ and washed with phosphate-buffered saline. The cells were then treated with 50 $\mu$ l DNA Prep LPR (Beckman Coulter, Fullerton, CA, USA) for $30 \mathrm{~min}$, after which $500 \mu \mathrm{l}$ DNA Prep Stain (Beckman Coulter) was added and the cells were incubated for an additional $30 \mathrm{~min}$ at room temperature. Flow cytometric analysis (Becton-Dickinson, Franklin Lakes, NJ, USA) was performed to examine the cell-cycle distribution.

Caspase-3 activity. HCT 116, HT-29, and SW480 cells $\left(5 \times 10^{3}\right.$ cells/well $)$ were seeded in 96 -well plates. The plates were removed from the incubator $48 \mathrm{~h}$ after adenoviral infection and equilibrated to room temperature. Caspase Glo 3/7 reagent (100 $\mu$; Promega) was then added to each well and the plates were incubated at room temperature for $1 \mathrm{~h}$, after which the luminescence of each sample was measured with a luminometer.

Statistical analysis. All data are displayed as the mean \pm SD. Densitometric analysis of the protein and mRNA levels was achieved using Image-Pro Plus 5.0.2 software (Media Cybernetics, Bethesda, MD, USA). The statistical difference between the three groups was analysed by the Student-Newman-Keuls test for multiple comparisons. All statistical analyses were performed using SPSS 16.0 for Windows (SPSS, Chicago, IL, USA). A P-value $<0.05$ was considered statistically significant.

\section{Results}

Mfn2 expression in CRC tissues is significantly lower compared to that in adjacent normal tissues. Mfn2 mRNA and protein were detected in all 15 cases of CRC specimen (Fig. 1 and data not shown). Mfn2 protein and DNA levels were both decreased in the CRC tissues compared to that in 

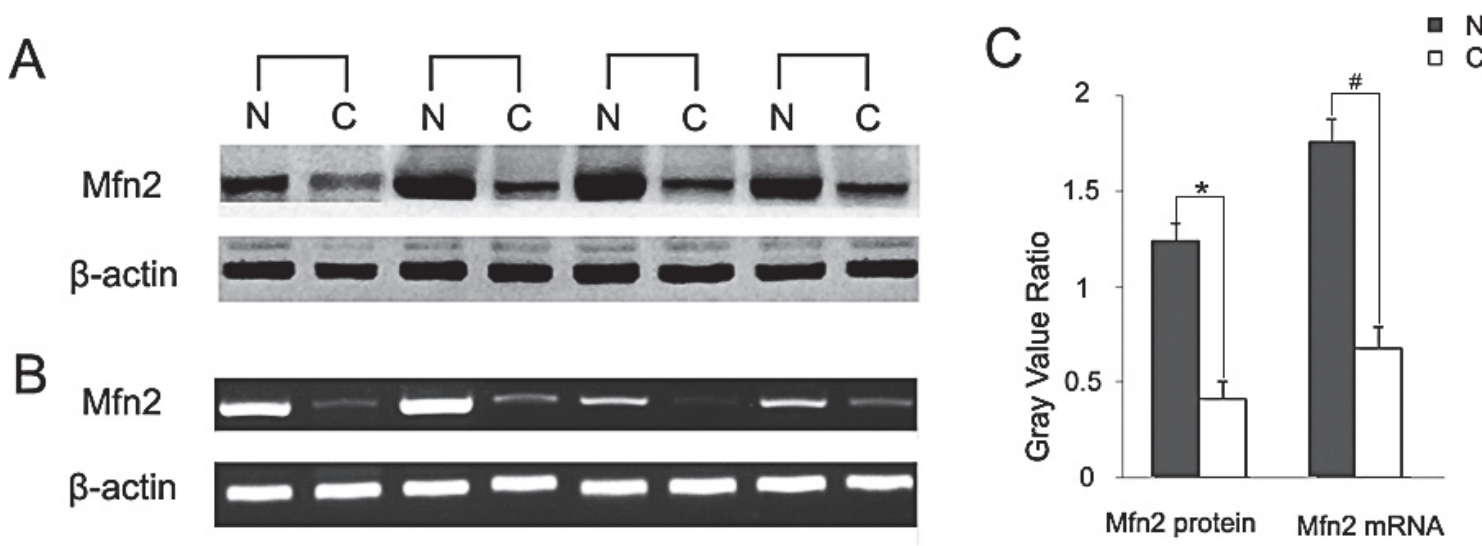

Figure 1. Mitofusin-2 (Mfn2) expression in colorectal cancer (CRC) tissues and corresponding adjacent normal tissues. (A) Representative Western blot results for four paired samples are shown. $\beta$-actin was used as a loading control. (B) Corresponding Mfn2 mRNA level as shown using RT-PCR. (C) Densitometric analysis of the Mfn2 protein and DNA levels (mean \pm SD) compared with $\beta$-actin in CRC and corresponding adjacent normal tissues $\left({ }^{*} \mathrm{P}<0.01 ; \# \mathrm{P}<0.05\right)$. N, corresponding non-tumorous tissues; $\mathrm{C}, \mathrm{CRC}$ tissues; $\mathbf{n}=15$.

the non-tumorous tissues $\left(124.7 \pm 77.4\right.$ vs. $47.6 \pm 28.3,{ }^{*} \mathrm{P}<0.01$; $168.9 \pm 130.7$ vs. $71.6 \pm 46.2, \# \mathrm{P}<0.05)$.

Increased expression of Mfn2 mediated by an adenoviral vector in CRC cells and its effect on cancer cell proliferation.
HCT 116, HT-29, and SW480 cells infected with Ad-Mfn2 expressed much more Mfn2 protein than did those infected with Ad-GFP (Fig. 2A), which indicates that Ad-Mfn2 caused efficient Mfn2 overexpression.

A

HCT 116

HT-29

SW480

Mock Ad-GFP Ad-Mfn2

Mock Ad-GFP Ad-Mfn2

Mock Ad-GFP Ad-Mfn2

Mfn2
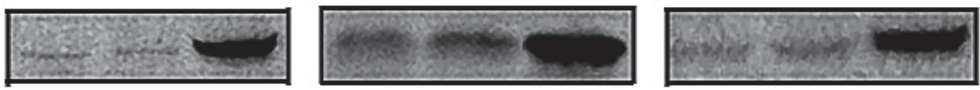

$\beta$-actin
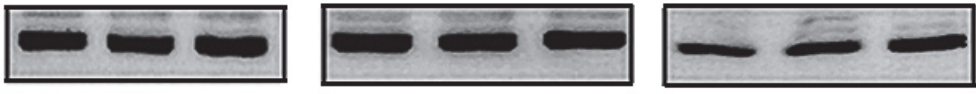

B

HCT 116

HT-29

SW480
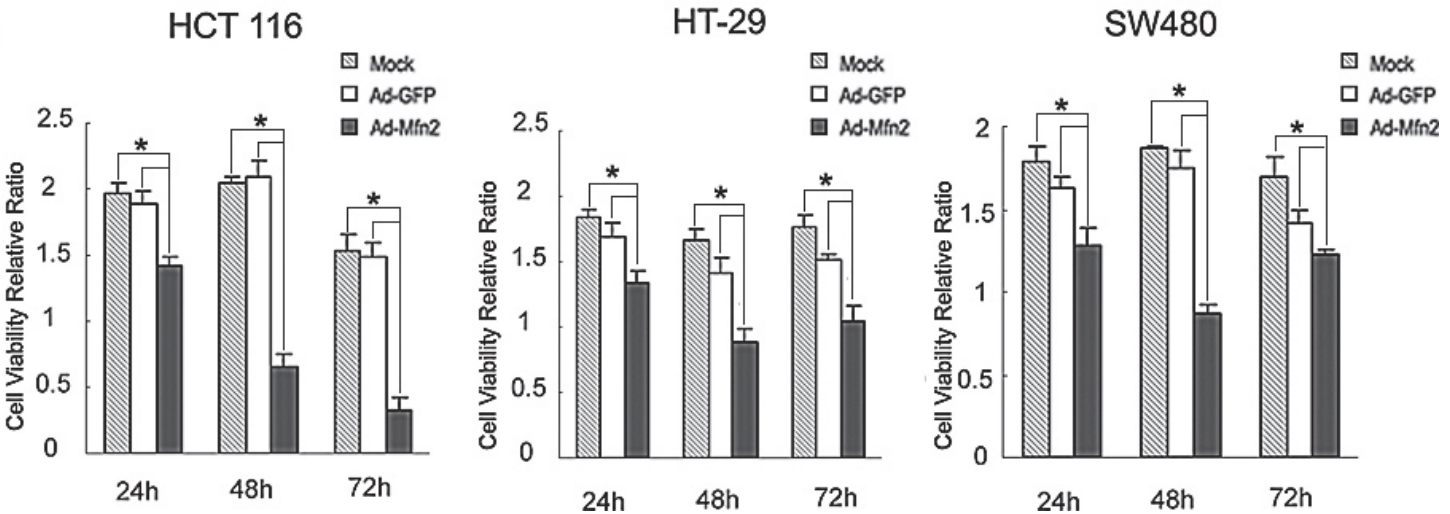

Figure 2. Adenovirus-mediated mitofusin-2 (Mfn2) overexpression in colorectal cancer (CRC) cells and its effect on cancer cell proliferation. (A) CRC cells were infected with an adenoviral vector encoding green fluorescent protein (Ad-GFP) or the complete Mfn2 open reading frame (Ad-Mfn2) at a multiplicity of infection (MOI) of 100. After a 48-h incubation, $\mathrm{Mfn} 2$ expression was analysed by Western blotting. $\beta$-actin was included as a loading control. (B) HCT 116, HT-29, and SW480 cells were uninfected (mock) or infected with 100 MOI of Ad-GFP or Ad-Mfn2. Cell viability was analysed at 24,48 , and $72 \mathrm{~h}$ post-infection using a WST-8 dye cell viability analyser $\left(\mathrm{n}=6 ;{ }^{\star} \mathrm{P}<0.005\right)$. 
To examine the anti-proliferative effect of Mfn2 overexpression, HCT 116, HT-29, and SW480 cells were infected with Ad-Mfn2 or Ad-GFP, and cell viability was analysed at 24, 48, and $72 \mathrm{~h}$ after infection. As shown in Fig. 2B, Ad-Mfn2 infection significantly inhibited the proliferation of CRC cells compared with Ad-GFP.

Mfn2 overexpression induces a cell cycle arrest in CRC cells at G2/M phase. To determine the potential mechanism responsible for the Mfn2 overexpression-mediated inhibition of CRC cell proliferation, we examined the cell-cycle distribution by flow cytometry. An altered distribution in response to increased Mfn2 expression was observed (Fig. 3). After $24 \mathrm{~h}$ of exposure to $10 \% \mathrm{FBS}$, a significant increase in G2/Mphase cells was noted in Ad-Mfn2-infected HCT 116, HT-29, and SW480 cells compared to uninfected cells and Ad-GFPinfected cells, and this was accompanied by a synchronous decrease in the percentage of G1-phase cells.
Then,we used immunoblot analysis to examine the effects of Ad-Mfn2 on the expression levels of G2/M cell cycle-related proteins, including $\mathrm{p}-\mathrm{Cdc} 2$ (Tyr 15), cyclin B1, Myt1, p-Wee1 (Ser642), and p21, in CRC cells. Our results indicate increased levels of p-cdc2 (Tyr15) and Myt1 but a decreased level of cyclin B1 in CRC cells infected with Ad-Mfn2 after 48 h (Fig. 4). No obvious change in the level of $\mathrm{p}$-Wee1, or $\mathrm{p} 21$ was detected (data not shown). To further delineate the cellular and molecular mechanisms underlying the Ad-Mfn2-induced growth arrest, we evaluated the possible regulatory role of Mfn2 in ERK1/2 signalling. Mfn2 overexpression resulted in an obvious reduction in p-ERK1/2 protein expression (Fig. 4).

Mfn2 overexpression triggers apoptosis in CRC cells. To further explore the effect of Ad-Mfn2 in apoptosis, we assessed caspase- 3 activity $48 \mathrm{~h}$ after infection with Ad-Mfn2 or AdGFP, and in mock-infected cells using the Caspase Glo 3/7 assay system. Caspase-3 activity was significantly increased

\section{HCT 116}
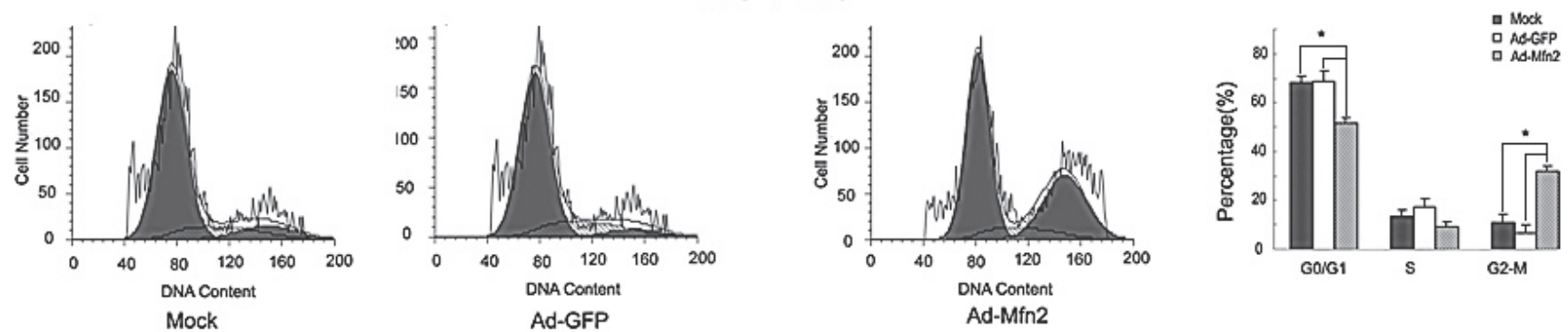

HT-29
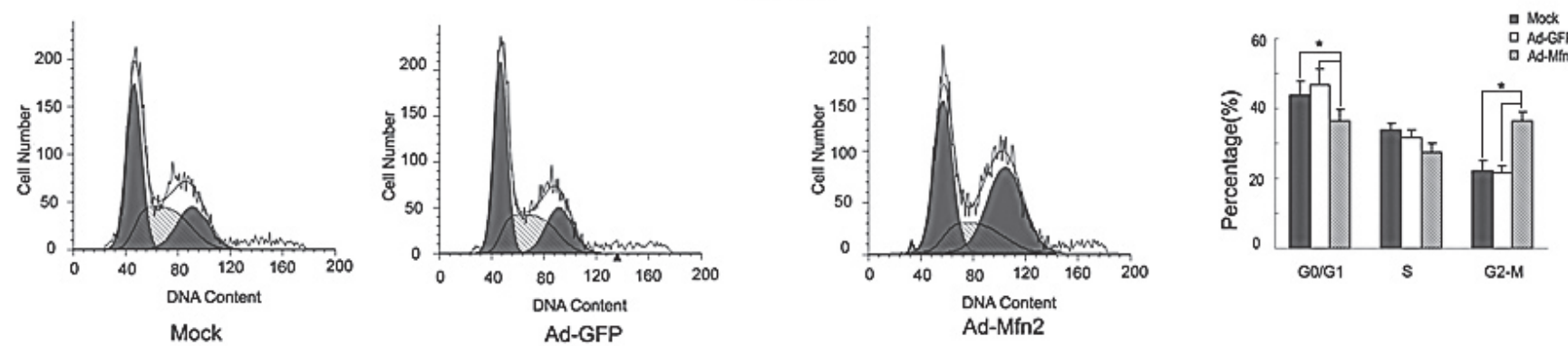

\section{SW480}
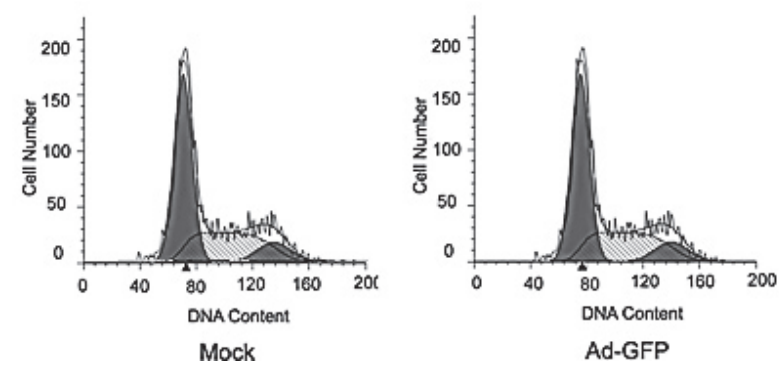
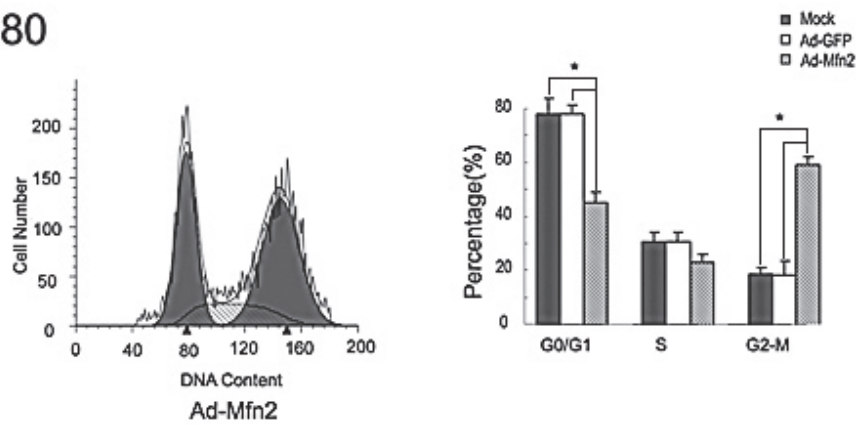

Figure 3. Overexpressed mitofusin-2 (Mfn2)-induced cell-cycle arrest in colorectal cancer (CRC) cells. Representative examples of the cell-cycle distribution in uninfected (mock), Ad-GFP), and Ad-Mfn2 groups. CRC cells were first synchronised and then infected with either Ad-GFP or Ad-Mfn2 at $100 \mathrm{pfu} /$ cell. Next, the cells were maintained in $\mathbf{0 . 2 \%}$ foetal bovine serum for $\mathbf{2 4} \mathrm{h}$ and then stimulated with normal serum medium. Flow cytometric analysis was used to examine the cell-cycle distribution $\left(\mathrm{n}=3 ;{ }^{\star} \mathbf{P}<0.01\right.$, compared with uninfected or Ad-GFP). 


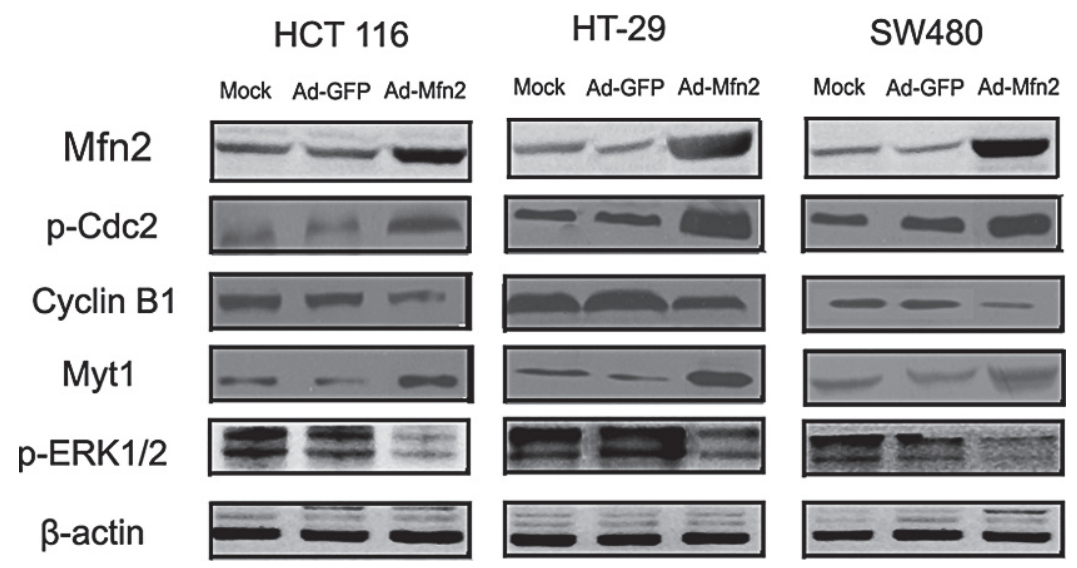

Figure 4. Overexpressed mitofusin-2 (Mfn2) effect on p-cdc2, cyclin B1, Myt1, and p-ERK1/2 protein levels in colorectal cancer (CRC) cells. HCT 116, HT-29, and SW480 cells were infected with adenoviral vector green fluorescent protein (Ad-GFP) or Ad-Mfn2 at a multiplicity of infection of 100 . After a 48-h incubation, p-cdc2, cyclin B1, Myt1, and p-ERK1/2 protein levels were analysed by Western blotting. $\beta$-actin was included as a loading control.

in HCT 116, HT-29, and SW480 cells infected with Ad-Mfn2 compared to both the Ad-GFP and mock-infected groups. (Fig. 5A). PARP and cleaved caspase-3 were further detected by Western blotting in CRC cells $48 \mathrm{~h}$ after infection. Ad-Mfn2 infection resulted in increased cleaved caspase- 3 and cleaved PARP expression (Fig. 5B). This indicates that the caspase- 3 effector was activated during Ad-Mfn2-induced apoptosis.

\section{Discussion}

$\mathrm{CRC}$ is a major public health problem in developed and undeveloped countries $[1,11]$. CRC develops as a result of the progressive accumulation of genetic alterations that transform normal colonic epithelial cells into colon adenocarcinoma cells [12]. Despite recent advances in our understanding of the CRC carcinogenic process, the need for further understanding the molecular mechanism of CRC is urgent. In this study, we found that Mfn 2 overexpression provided anti- proliferative efficacy against CRC cells. We first confirmed that the overexpression of Mfn2 induced a G2/M-phase arrest in CRC cells.

The gene encoding Mfn2, which has been mapped to chromosome 1q36.22, is expressed in various tissues, including the brain, heart, and skeletal muscle $[5,13]$. Abnormalities in chromosome 1q36 have been suggested to be an early event in the development of human cancers, including CRC [14]. In the past few decades, many tumor suppressor genes have been found in this region [15-16]. Using Western blot analysis and RT-PCR analysis, we found that Mfn2 protein and mRNA levels were significantly lower in CRC tissues than in corresponding normal tissues. Overexpression of $\mathrm{Mfn} 2$ protein
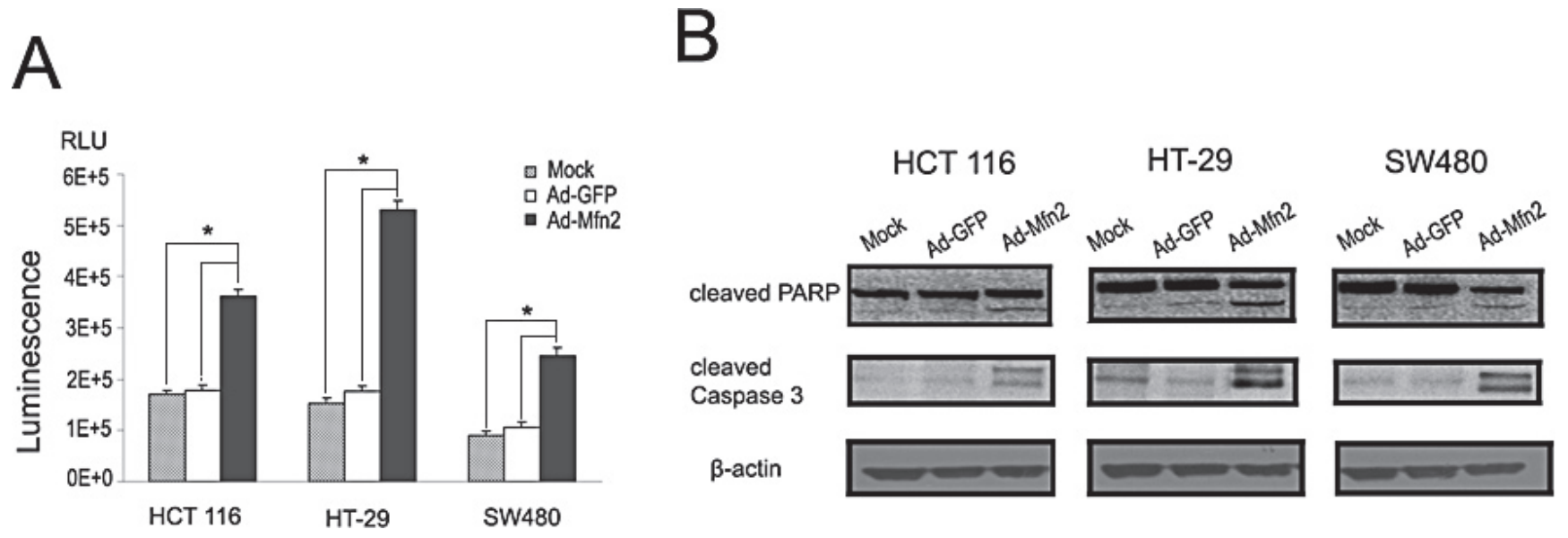

Figure 5. Mitofusin-2 (Mfn2) overexpression-induced apoptosis in colorectal cancer (CRC) cells. (A) Caspase-3 activity was increased in CRC cells transfected with adenoviral vector (Ad)-Mfn2 compared with Ad-green fluorescent protein (GFP)- and mock-transfected cells $\left(48 \mathrm{~h}, \mathrm{n}=6 ;{ }^{*} \mathrm{P}<0.001\right)$. (B) Mfn2 overexpression in CRC cells infected with Ad-Mfn2 resulted in increased protein levels of PARP cleavage and cleaved caspase-3, as shown by Western blotting. $\beta$-actin was included as a loading control. 
mediated by adenovirus has a significant anti-proliferative effect in a wide range of cancer cell lines [8]. Our results indicate that Ad-Mfn2 also had a significant anti-proliferative effect in HCT116, HT-29, and SW480 cells. Ad-Mfn2 mediated Mfn2 overexpression, prevented proliferation, and promoted apoptosis in CRC cells. The overexpression of Mfn2 protein results in caspase- 3 and -9 activation in VSMCs and is mediated by the mitochondrial apoptotic pathway $[7,17,18]$. Our current findings show that caspase- 3 activity increased significantly in HCT 116, HT-29, and SW480 cells infected with Ad-Mfn2 compared with Ad-GFP and mock-infected cells, and that the protein levels of cleaved caspase- 3 and cleaved PARP were significantly increased.

The cell cycle is a series of events leading to cell division. Cell-cycle retardation is an important anti-proliferative mechanism in various cancers. Rigorous quality control events, or checkpoints, ensure the proper progression of cells through the cell cycle and allow cells to respond to DNA damage. Eukaryotic cell-cycle checkpoints are controlled by the sequential activation of a group of Ser-Thr kinases called cyclin-dependent kinases (Cdks), which trigger the transition to subsequent phases of the cycle [19-20]. Cancer cells are mainly dependent on the G2 checkpoint to repair DNA damage, due to the presence of defective G1 checkpoint mechanisms, and the S-phase checkpoint facilitates slowing rather than causing a complete cell cycle arrest [21]. Most cell cycle regulation is dependent on phosphorylation states. pcdc2, or Cdk1, which was originally identified in fission yeast, is the prototypical cdk [22]. p-cdc2 contains inhibitory and stimulatory phosphorylation sites. The phosphorylation of $\mathrm{p}$ cdc2 at Tyr15 and Thr14, performed by the kinases Weel and Mytl, prevents kinase activity [22]. Flow cytometric analysis showed that Mfn2 overexpression mediated a G2/M-phase arrest and inhibited cell growth in HCT 116, HT-29, and SW480 cells. We confirmed this result by Western blotting and showed that $48 \mathrm{~h}$ after the infection of CRC cells with Ad-Mfn2 the levels of p-cdc2 (Tyr15) and Myt1 increased while the level of cyclin B1 decreased. We did not observe an obvious change in the level of p-Wee1, or p21 (data not shown), but an obvious reduction in the level of p-ERK1/2 induced by Mfn 2 overexpression. ERK1/2 is activated during the G2/M phase of the cell cycle, whereas it is dephosphorylated in metaphase-arrested cells [23]. The cyclin B/Cdk1 complex is retained in the cytosol, leading to weak Cdk1 activation and the impaired entry of cells into mitosis when ERK1/2 activity is inhibited [24]. We demonstrated previously that Mfn2 is a novel p53-inducible target gene [9]. Thus, we speculate that Mfn2 inhibits the growth of CRC cells: the role of ERK1/2,G2/M-phase cell cycle arrest and p53.

In summary, our results confirm that Mfn2 is a hyperplasia suppressor gene in CRC cells. Mfn2 was downregulated in CRC tissues, compared with nearby non-tumorous tissues. Mfn2 overexpression, mediated by an adenoviral vector, exerted an anti- proliferative effect by inducing a G2/M-phase arrest in CRC cells.
Acknowledgements: This work was supported by grants from the National Natural Science Foundation of China (No. 30901787), the Program of the Science and Technology Bureau of Zhejiang Province (No. 2010R10072).

\section{References}

[1] LEVIN AA. Patient safety: rejecting the status quo. N C Med J 2005; 66: 91-95.

[2] JENKINSON F, STEELE RJ. Colorectal cancer screening - methodology. Surgeon 2010; 8: 164-171. http: //dx.doi. org/10.1016/j.surge.2009.10.015

[3] SAIF MW, CHU E. Biology of colorectal cancer. Cancer J 2010; 16: 196-201. http: //dx.doi.org/10.1097/ PPO.0b013e3181e076af

[4] SHAW P, COSTA J. Molecular biology of colon cancer (review). Anticancer Res 1989; 9: 21-27.

[5] CHEN KH, GUO X, MA D, GUO Y, LI Q, et al. Dysregulation of HSG triggers vascular proliferative disorders. Nat Cell Biol 2004; 6: 872-883. http: //dx.doi.org/10.1038/ncb1161

[6] KARBOWSKI M, NORRIS KL, CLELAND MM, JEONG SY, YOULE RJ. Role of Bax and Bak in mitochondrial morphogenesis. Nature 2006; 443: 658-662. http: //dx.doi. org/10.1038/nature05111

[7] KARBOWSKI M, LEE YJ, GAUME B, JEONG SY, FRANK $S$, et al. Spatial and temporal association of Bax with mitochondrial fission sites, Drp1, and Mfn2 during apoptosis. J Cell Biol 2002; 159: 931-938. http: //dx.doi.org/10.1083/ jcb.200209124

[8] WU L, LI Z, ZHANG Y, ZHANG P, ZHU X, et al. Adenovirus-expressed human hyperplasia suppressor gene induces apoptosis in cancer cells. Mol Cancer Ther 2008; 7: 222-232. http: //dx.doi.org/10.1158/1535-7163.MCT-07-0382

[9] WANG W, CHENG X, LU J, WEI J, FU G, et al. Mitofusin2 is a novel direct target of p53. Biochem Biophys Res Commun 2010; 400: 587-592. http: //dx.doi.org/10.1016/ j.bbrc. 2010.08 .108

[10] WANG W, ZHU F, WANG S, WEI J, JIA C, et al. HSG provides antitumor efficacy on hepatocellular carcinoma both in vitro and in vivo. Oncol Rep 2010; 24: 183-188.

[11] JEMAL A, MURRAY T, WARD E, SAMUELS A, TIWARI RC, et al. Cancer statistics, 2005. CA Cancer J Clin 2005; 55: 10-30. http: //dx.doi.org/10.3322/canjclin.55.1.10

[12] WHITE RL. Colon cancer. Molecular biology of the APC protein. Pathol Biol (Paris) 1997; 45: 240-244.

[13] BACH D, PICH S, SORIANO FX, VEGA N, BAUMGARTNER $B$, et al. Mitofusin-2 determines mitochondrial network architecture and mitochondrial metabolism. A novel regulatory mechanism altered in obesity. J Biol Chem 2003; 278: 17190-17197. http: //dx.doi.org/10.1074/jbc.M212754200

[14] BAGCHI A, MILLS AA. The quest for the 1 p36 tumor suppressor. Cancer Res 2008; 68: 2551-2556. http: //dx.doi. org/10.1158/0008-5472.CAN-07-2095

[15] MORI N, MOROSETTI R, SPIRA S, LEE S, BEN-YEHUDA $\mathrm{D}$, et al. Chromosome band 1p36 contains a putative tumor suppressor gene important in the evolution of chronic myelocytic leukemia. Blood 1998; 92: 3405-3409. 
[16] SMEDLEY D, SIDHAR S, BIRDSALL S, BENNETT D, HERLYN M, et al. Characterization of chromosome 1 abnormalities in malignant melanomas. Genes Chromosomes Cancer 2000; 28: 121-125. http: //dx.doi.org/10.1002/(SICI)10982264(200005)28: 1<121: : AID-GCC14>3.0.CO; 2-O

[17] CHEN H, DETMER SA, EWALD AJ, GRIFFIN EE, FRASER SE, et al. Mitofusins Mfn1 and Mfn2 coordinately regulate mitochondrial fusion and are essential for embryonic development. J Cell Biol 2003; 160: 189-200. http: //dx.doi. org/10.1083/jcb.200211046

[18] GUO X, CHEN KH, GUO Y, LIAO H, TANG J, et al. Mitofusin 2 triggers vascular smooth muscle cell apoptosis via mitochondrial death pathway. Circ Res 2007; 101: 1113-1122. http: //dx.doi.org/10.1161/CIRCRESAHA.107.157644

[19] SHERR CJ. Cancer cell cycles. Science 1996; 274: 1672-1677. http: //dx.doi.org/10.1126/science.274.5293.1672
[20] MURRAY AW. Recycling the cell cycle: cyclins revisited. Cell 2004; 116: 221-234. http: //dx.doi.org/10.1016/S00928674(03)01080-8

[21] BUCHER N, BRITTEN CD. G2 checkpoint abrogation and checkpoint kinase-1 targeting in the treatment of cancer. Br J Cancer 2008; 98: 523-528. http: //dx.doi.org/10.1038/ sj.bjc. 6604208

[22] STARK GR, TAYLOR WR. Analyzing the G2/M checkpoint. Methods Mol Biol 2004; 280: 51-82.

[23] TAMEMOTO H, KADOWAKI T, TOBE K, UEKI K, IZUMI $\mathrm{T}$, et al. Biphasic activation of two mitogen-activated protein kinases during the cell cycle in mammalian cells. J Biol Chem 1992; 267: 20293-20297.

[24] CHAMBARD JC, LEFLOCH R, POUYSSEGUR J, LENORMAND P. ERK implication in cell cycle regulation. Bba-Mol Cell Res 2007; 1773: 1299-1310. 